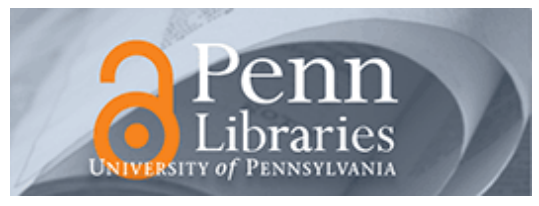

University of Pennsylvania ScholarlyCommons

\title{
$5-1998$
}

\section{An Optimal Acceptance Policy for an Urn Scheme}

Robert W. Chen

Alan Zame

Andrew M. Odlyzko

Larry A. Shepp

University of Pennsylvania

Follow this and additional works at: https://repository.upenn.edu/statistics_papers

Part of the Discrete Mathematics and Combinatorics Commons, and the Statistics and Probability Commons

\section{Recommended Citation}

Chen, R. W., Zame, A., Odlyzko, A. M., \& Shepp, L. A. (1998). An Optimal Acceptance Policy for an Urn Scheme. SIAM Journal on Discrete Mathematics, 11 (2), 183-195. http://dx.doi.org/10.1137/ S0895480195282148

This paper is posted at ScholarlyCommons. https://repository.upenn.edu/statistics_papers/416

For more information, please contact repository@pobox.upenn.edu. 


\title{
An Optimal Acceptance Policy for an Urn Scheme
}

\author{
Abstract \\ An urn contains $m$ balls of value -1 and $p$ balls of value +1 . At each turn a ball is drawn randomly, without \\ replacement, and the player decides before the draw whether or not to accept the ball, i.e., the bet where \\ the payoff is the value of the ball. The process continues until all $m+p$ balls are drawn. Let $V(m, p)$ denote \\ the value of this acceptance $(m, p)$ urn problem under an optimal acceptance policy. In this paper, we first \\ derive an exact closed form for $\mathrm{V}(m, p)$ and then study its properties and asymptotic behavior. We also \\ compare this acceptance $(m, p)$ urn problem with the original $(m, p)$ urn problem which was introduced by \\ Shepp [Ann. Math. Statist., 40 (1969), pp. 993--1010]. Finally, we briefly discuss some applications of this \\ acceptance $(m, p)$ urn problem and introduce a Bayesian approach to this optimal stopping problem. \\ Some numerical illustrations are also provided.

\section{Keywords} \\ optimal stopping, acceptance policy, urn models, Bayesian approach \\ Disciplines \\ Discrete Mathematics and Combinatorics | Statistics and Probability
}




\title{
AN OPTIMAL ACCEPTANCE POLICY FOR AN URN SCHEME*
}

\author{
ROBERT W. $\mathrm{CHEN}^{\dagger}$, ALAN ZAME ${ }^{\dagger}$, ANDREW M. ODLYZKO ${ }^{\ddagger}$, AND LARRY A. SHEPP $\ddagger$
}

\begin{abstract}
An urn contains $m$ balls of value -1 and $p$ balls of value +1 . At each turn a ball is drawn randomly, without replacement, and the player decides before the draw whether or not to accept the ball, i.e., the bet where the payoff is the value of the ball. The process continues until all $m+p$ balls are drawn. Let $\bar{V}(m, p)$ denote the value of this acceptance $(m, p)$ urn problem under an optimal acceptance policy. In this paper, we first derive an exact closed form for $\bar{V}(m, p)$ and then study its properties and asymptotic behavior. We also compare this acceptance $(m, p)$ urn problem with the original $(m, p)$ urn problem which was introduced by Shepp [Ann. Math. Statist., 40 (1969), pp. 993-1010]. Finally, we briefly discuss some applications of this acceptance $(m, p)$ urn problem and introduce a Bayesian approach to this optimal stopping problem. Some numerical illustrations are also provided.
\end{abstract}

Key words. optimal stopping, acceptance policy, urn models, Bayesian approach

AMS subject classifications. primary, 60G40; secondary, 60K99

PII. S0895480195282148

1. Introduction. In [7], Shepp considered the following optimal stopping problem: An $(m, p)$ urn contains $m$ balls of value -1 and $p$ balls of value +1 , and the player is allowed to draw balls randomly, without replacement, until he wants to stop. Shepp was interested in finding, for what $m$ and $p$, if there is an optimal drawing policy for which $V(m, p)$ is positive, where $V(m, p)$ is the value of this $(m, p)$ urn problem under an optimal drawing policy. In particular, he showed that for every positive integer $p$ there is a positive integer $\beta(p)$ for which $V(m, p)>0$ or $=0$, with $0 \leq m \leq \beta(p)$ or $m>\beta(p)$ accordingly. In [2,3], Boyce, motivated by applications to financial and marketing problems, also studied this $(m, p)$ urn problem. In [4], Chen and Hwang derived some new properties of $V(m, p)$ that give additional insight into the structure of the optimal drawing policy for this $(m, p)$ urn problem.

In this paper, we study a new $(m, p)$ urn problem that we call an acceptance $(m, p)$ urn problem and that can be simply described as follows: An urn contains $m$ balls of value -1 and $p$ balls of value +1 . At each turn a ball is drawn randomly, without replacement, and the player decides before the draw whether or not to accept the ball, i.e., the bet where the payoff is the value of the ball. The process will continue until all $m+p$ balls are drawn. We are interested in the value $\bar{V}(m, p)$ of this acceptance $(m, p)$ urn problem under an optimal acceptance policy. We first derive an exact closed form for $\bar{V}(m, p)$ by a simple probabilistic argument and obtain inequalities of the form $\bar{V}(m, p)<\bar{V}(m+1, p+1)$, in the spirit of [3] and [4] for the original urn problem. Then we study the asymptotic behavior of $\bar{V}(m, p)$. We also compare this acceptance $(m, p)$ urn problem with the original $(m, p)$ urn problem. Finally, we briefly indicate an application of this acceptance urn version of the optimal policy problematics to (in-and-out) bond trading and introduce a Bayesian approach to this optimal stopping problem. Some numerical illustrations are also provided.

* Received by the editors February 27, 1995; accepted for publication (in revised form) January 30, 1997.

http://www.siam.org/journals/sidma/11-2/28214.html

${ }^{\dagger}$ Department of Mathematics and Computer Science, University of Miami, Coral Gables, FL 33124 (chen@cs.miami.edu, zame@cs.miami.edu).

${ }_{\ddagger}$ AT\&T Bell Laboratories, 600 Mountain Avenue, Murray Hill, NJ 07974 (amo@research.att.com, las@research.att.com). 
2. Exact solutions of $\overline{\boldsymbol{V}}(\boldsymbol{m}, \boldsymbol{p})$. For each nonnegative integer $m$ and $p$ such that $m+p \geq 1$, let $A(m, p)$ be the expected value of accepting the current drawn ball from the $(m, p)$ urn, assuming an optimal acceptance policy is followed after the current draw, and let $N(m, p)$ be the expected value of not accepting the current drawn ball from the $(m, p)$ urn, assuming an optimal acceptance policy is followed after the current draw. It is clear that $\bar{V}(m, p)=\max \{A(m, p), N(m, p)\}, A(m, p)=$ $(p /(m+p))(1+\bar{V}(m, p-1))+(m /(m+p))(-1+\bar{V}(m-1, p))$, and $N(m, p)=(p /(m+$ $p)) \bar{V}(m, p-1)+(m /(m+p)) \bar{V}(m-1, p)$. Hence $A(m, p)=(p-m) /(m+p)+N(m, p)$. Therefore, $\bar{V}(m, p)=A(m, p)$ if $p \geq m$ and $\bar{V}(m, p)=N(m, p)$ if $p<m$. The optimal acceptance policy can now be easily stated as follows: Accept the current drawn ball if the number of +1 balls is greater than or equal to the number of -1 balls, otherwise, do not accept the current drawn ball.

Based on the optimal acceptance policy, we will accept the drawn balls until the number of +1 balls is less than the number of -1 balls. Since the probability that starting from the position $(m, p)(m \neq p)$ and reaching the position $(i, i)(i>$ 0 and $i \leq \min \{m, p\})$ the first time is exactly equal to the probability of starting from the position $(p, m)$ and reaching the position $(i, i)$ the first time, it is easy to see that the following two theorems hold.

THEOREM 2.1. For any nonnegative integer $m$ and $p,|\bar{V}(m, p)-\bar{V}(p, m)|=$ $|m-p|$.

THEOREM 2.2. If $m>p$,

$$
\begin{aligned}
\bar{V}(m, p)= & \sum_{j=1}^{p} \bar{V}(j, j)\left\{\left(\begin{array}{c}
m+p-2 j-1 \\
m-j-1
\end{array}\right)\right. \\
& \left.-\left(\begin{array}{c}
m+p-2 j-1 \\
m-j
\end{array}\right)\right\} \frac{p \cdots(j+1) m \cdots(j+1)}{(p+m)(p+m-1) \cdots(2 j+1)} \\
= & \sum_{j=1}^{p} D(j, j) \frac{(m-p)}{(m+p-2 j)} \frac{\left(\begin{array}{c}
m+p-2 j \\
m-j
\end{array}\right)}{\left(\begin{array}{c}
m+p \\
p
\end{array}\right)} .
\end{aligned}
$$

Here, $D(i, j)=\left(\begin{array}{c}i+j \\ j\end{array}\right) \bar{V}(i, j)$.

THEOREM 2.3. For any positive integer $m \geq p$,

$$
\begin{gathered}
\bar{V}(m, p)=\sum_{i=1}^{p} \frac{\left(\begin{array}{c}
m+p-2 i \\
p-i
\end{array}\right)\left(\begin{array}{c}
2 i \\
i
\end{array}\right)}{2\left(\begin{array}{c}
m+p \\
p
\end{array}\right)}=\sum_{i=0}^{p-1} \frac{\left(\begin{array}{c}
m+p \\
i
\end{array}\right)}{\left(\begin{array}{c}
m+p \\
p
\end{array}\right)} \\
=p 2^{m+p} \int_{0}^{\frac{1}{2}} x^{m}(1-x)^{p-1} d x, \\
\text { and } \bar{V}(m, m)=\left(2^{2 m-1} /\left(\begin{array}{c}
2 m \\
m
\end{array}\right)\right)-\frac{1}{2} .
\end{gathered}
$$

Proof. Let $X_{i}$ be the value of the $i$ th ball $(i=1,2, \ldots, m+p)$, and let $S_{k}=$ $\sum_{i=k+1}^{m+p} X_{i}$ be the $k$ th (tail) partial sum $(k=0,1,2, \ldots, m+p-1)$. Let $N=\#\{k$ : $\left.S_{k}=0,0 \leq k<m+p\right\}$. Notice that $P\left(S_{k+1}=1 \mid S_{k}=0\right)=1 / 2$ and that whenever $S_{j}=1$, the player gains one unit (according to the optimal policy) by time $\tau$, where $\tau=\min \left\{k \mid k>j\right.$ and $\left.S_{k}=0\right\}$. Hence, $\bar{V}(m, p)=1 / 2 E(N)$. Notice that each realization of this urn problem is an arrangement of $m$ identical -1 balls and $p$ identical +1 balls and that each realization occurs with probability $1 /\left(\begin{array}{c}m+p \\ p\end{array}\right)$. Thus, $\left(\begin{array}{c}m+p \\ p\end{array}\right) E(N)=\sum_{w} N(w)$, where the sum is taken over all realizations $w$. Next let $T_{i}$ 
be the number of realizations in which $S_{m+p-2 i}=0$. Since $\sum_{w} N(w)=\sum_{i=1}^{p} T_{i}$ and $T_{i}=\left(\begin{array}{c}m+p-2 i \\ p-i\end{array}\right)\left(\begin{array}{c}2 i \\ i\end{array}\right)$, we have $\left(\begin{array}{c}m+p \\ p\end{array}\right) E(N)=\sum_{i=1}^{p}\left(\begin{array}{c}m+p-2 i \\ p-i\end{array}\right)\left(\begin{array}{c}2 i \\ i\end{array}\right)$. Therefore,

$$
\bar{V}(m, p)=\frac{1}{2} E(N)=\sum_{i=1}^{p} \frac{\left(\begin{array}{c}
m+p-2 i \\
p-i
\end{array}\right)\left(\begin{array}{c}
2 i \\
i
\end{array}\right)}{2\left(\begin{array}{c}
m+p \\
p
\end{array}\right)} .
$$

By the combinatorial identity, $\sum_{i=1}^{p}\left(\begin{array}{c}m+p-2 i \\ p-i\end{array}\right)\left(\begin{array}{c}2 i \\ i\end{array}\right)=2 \sum_{i=0}^{p-1}\left(\begin{array}{c}m+p \\ i\end{array}\right)$; then

$$
\bar{V}(m, p)=\sum_{i=0}^{p-1} \frac{\left(\begin{array}{c}
m+p \\
i
\end{array}\right)}{\left(\begin{array}{c}
m+p \\
p
\end{array}\right)} .
$$

Since

$$
\sum_{i=0}^{l-1}\left(\begin{array}{c}
n \\
i
\end{array}\right)\left(\frac{1}{2}\right)^{n}=l\left(\begin{array}{c}
n \\
i
\end{array}\right) \int_{0}^{\frac{1}{2}} x^{n-l}(1-x)^{l-1} d x
$$

then

$$
\sum_{i=0}^{p-1} \frac{\left(\begin{array}{c}
m+p \\
i
\end{array}\right)}{\left(\begin{array}{c}
m+p \\
p
\end{array}\right)}=2^{m+p} p \int_{0}^{\frac{1}{2}} x^{m}(1-x)^{p-1} d x .
$$

By the combinatorial identity, $\sum_{i=1}^{m}\left(\begin{array}{c}2 m-2 i \\ m-i\end{array}\right)\left(\begin{array}{c}2 i \\ i\end{array}\right)=4^{m}-\left(\begin{array}{c}2 m \\ m\end{array}\right)$ [5, p. 32]; then

$$
\bar{V}(m, m)=\frac{2^{2 m-1}}{\left(\begin{array}{c}
2 m \\
m
\end{array}\right)}-\frac{1}{2} .
$$

The proof of Theorem 2.3 is now complete.

THEOREM 2.4. For any positive integer $m$ and $p, D(m, p)=\bar{V}(m, p)\left(\begin{array}{c}m+p \\ p\end{array}\right)$ is a positive integer.

Proof. By Theorem 2.1, it is sufficient to consider the case when $m \geq p$. By Theorem 2.3, $D(m, p)=\bar{V}(m, p)\left(\begin{array}{c}m+p \\ p\end{array}\right)=\sum_{i=0}^{p-1}\left(\begin{array}{c}m+p \\ i\end{array}\right)$ is a positive integer.

THEOREM 2.5. For any nonnegative integer $m$ and $p, \bar{V}(m+1, p+1)>\bar{V}(m, p)$.

Proof. Since $\bar{V}(m+1,1)>\bar{V}(m, 0)=0$ for any nonnegative integer $m$, by Theorem 2.1 we can and do assume $m \geq p \geq 1$. By Theorem 2.3,

$$
\begin{aligned}
& \bar{V}(m+1, p+1)-\bar{V}(m, p) \\
& =2^{m+p} \int_{0}^{\frac{1}{2}} x^{m}(1-x)^{p-1}(4(p+1) x(1-x)-p) d x \\
& =2^{m+p} \int_{0}^{\frac{1}{2}} x^{m-p+1}\left(4(p+1) x^{p}(1-x)^{p}-p x^{p-1}(1-x)^{p-1}\right) d x .
\end{aligned}
$$

It is sufficient to show that

$$
\int_{0}^{\frac{1}{2}} x^{m-p+1}\left(4(p+1) x^{p}(1-x)^{p}-p x^{p-1}(1-x)^{p-1}\right) d x>0 .
$$

Notice that

$$
\begin{aligned}
& \int_{0}^{\frac{1}{2}}\left(4(p+1) x^{p}(1-x)^{p}-p x^{p-1}(1-x)^{p-1}\right) d x \\
& =\frac{1}{2} \int_{0}^{1}\left(4(p+1) x^{p}(1-x)^{p}-p x^{p-1}(1-x)^{p-1}\right) d x=\frac{2 p !(p+1) !}{(2 p+1) !}-\frac{p ! p !}{(2 p) !}>0 .
\end{aligned}
$$


Let $x^{*}$ be the number in $(0,1 / 2)$ such that $4(p+1) x^{*}\left(1-x^{*}\right)=p$. Then $4(p+1) x(1-$ $x)-p \leq 0$ if $0 \leq x \leq x^{*}$ and $4(p+1) x(1-x)-p \geq 0$ if $x^{*} \leq x \leq 1 / 2$. Hence,

$$
\begin{aligned}
\int_{0}^{\frac{1}{2}} & \left(4(p+1) x^{p}(1-x)^{p}-p x^{p-1}(1-x)^{p-1}\right) d x \\
& =\int_{x^{*}}^{\frac{1}{2}}\left(4(p+1) x^{p}(1-x)^{p}-p x^{p-1}(1-x)^{p-1}\right) d x \\
& -\int_{0}^{x^{*}}\left(p x^{p-1}(1-x)^{p-1}-4(p+1) x^{p}(1-x)^{p}\right) d x>0 ;
\end{aligned}
$$

that is,

$$
\begin{aligned}
\int_{x^{*}}^{\frac{1}{2}} & \left(4(p+1) x^{p}(1-x)^{p}-p x^{p-1}(1-x)^{p-1}\right) d x \\
& >\int_{0}^{x^{*}}\left(p x^{p-1}(1-x)^{p-1}-4(p+1) x^{p}(1-x)^{p}\right) d x .
\end{aligned}
$$

By the Mean Value theorem,

$$
\begin{aligned}
\int_{0}^{\frac{1}{2}} & x^{m-p+1}\left(4(p+1) x^{p}(1-x)^{p}-p x^{p-1}(1-x)^{p-1}\right) d x \\
& =x_{2}^{m-p+1} \int_{x^{*}}^{\frac{1}{2}}\left(4(p+1) x^{p}(1-x)^{p}-p x^{p-1}(1-x)^{p-1}\right) d x \\
& -x_{1}^{m-p+1} \int_{0}^{x^{*}}\left(p x^{p-1}(1-x)^{p-1}-4(p+1) x^{p}(1-x)^{p}\right) d x
\end{aligned}
$$

where $0 \leq x_{1} \leq x^{*} \leq x_{2} \leq 1 / 2$. Hence,

$$
\int_{0}^{\frac{1}{2}} x^{m-p+1}\left(4(p+1) x^{p}(1-x)^{p}-p x^{p-1}(1-x)^{p-1}\right) d x>0
$$

since $m \geq p$. Therefore, $\bar{V}(m+1, p+1)>\bar{V}(m, p)$ for all nonnegative integers $m$ and $p$

THEOREM 2.6.

1. $1 /(m+p+1) \leq \bar{V}(m, p+1)-\bar{V}(m, p) \leq 1$.

2. $0 \leq \bar{V}(m, p)-\bar{V}(m+1, p) \leq 1-(1 /(m+p+1))$.

Proof. By Theorems 2.1 and 2.3 , it is easy to check that $\bar{V}(m, p)-\bar{V}(m+1, p) \geq 0$ and to also check that $1 /(m+p+1) \leq \bar{V}(m, p+1)-\bar{V}(m, p)$ is equivalent to that $\bar{V}(m, p)-\bar{V}(m+1, p) \leq 1-(1 /(m+p+1))$ by Theorem 2.1. Theorem 2.6 is clearly true when $n=m+p=1$. Now, by mathematical induction on $n$, we can prove Theorem 2.6 easily (details are omitted).

THEOREM 2.7. For any positive integer $k, \bar{V}(k m, m)$ and $\bar{V}(m, k m)$ are strictly increasing in $m$.

Proof. By Theorem 2.1, it is sufficient to show that $\bar{V}(k m, m)$ is strictly increasing in $m$. By Theorem 2.5, Theorem 2.7 holds when $k=1$. Now we will prove Theorem 2.7 when $k \geq 2$. By Theorem 2.3,

$$
\begin{aligned}
& \bar{V}(k(m+1), m+1)-\bar{V}(k m, m) \\
& \quad=2^{k m+m} \int_{0}^{\frac{1}{2}} x^{k m}(1-x)^{m-1}\left(2^{k+1}(m+1) x^{k}(1-x)-m\right) d x \\
& \quad=2^{k m+m} \int_{0}^{\frac{1}{2}} x^{m-1}(1-x)^{m-1} x^{k m-m+1}\left(2^{k+1}(m+1) x^{k}(1-x)-m\right) d x .
\end{aligned}
$$


Since $m \geq 1, x^{m-1}(1-x)^{m-1}$ is strictly increasing and nonnegative on the interval $[0,1 / 2], 2^{k+1}(m+1) x^{k}(1-x)-m \leq 0$ if $0 \leq x \leq x^{*}, \geq 0$ if $x * \leq x \leq 1 / 2$, where $0<x^{*}<1 / 2$. By the Mean Value theorem, it is sufficient to show that

$$
\int_{0}^{\frac{1}{2}} x^{k m-m+1}\left(2^{k+1}(m+1) x^{k}(1-x)-m\right) d x>0 .
$$

By a direct computation,

$$
\begin{aligned}
& \int_{0}^{\frac{1}{2}} x^{k m-m+1}\left(2^{k+1}(m+1) x^{k}(1-x)-m\right) d x \\
& \quad=(1 / 2)^{k m-m+2}\left(\frac{2(m+1)}{k m+k-m+2}-\frac{m+1}{k m+k-m+3}-\frac{m}{k m-m+2}\right)>0,
\end{aligned}
$$

since $k \geq 2$. Therefore, $\bar{V}(k(m+1), m+1)-\bar{V}(k m, m)>0$, and the proof of Theorem 2.7 now is complete.

3. Asymptotic behavior of $\overline{\boldsymbol{V}}(\boldsymbol{m}, \boldsymbol{p})$. By Theorems $2.1,2.2$, and 2.3, we have an exact closed form solution for $\bar{V}(m, p)$. However, it is only useful when $m$ or $p$ is small. In this section, we will derive some asymptotic forms for $\bar{V}(m, p)$ when $m$ and $p \rightarrow \infty$.

THEOREM 3.1. $\bar{V}(m, p) \rightarrow p /(m-p)$ if $m / p \rightarrow \lambda>1$.

Proof. By Theorem 2.3,

$$
\begin{aligned}
\bar{V}(m, p) & =\sum_{i=1}^{p} \frac{\left(\begin{array}{c}
m+p-2 i \\
p-i
\end{array}\right)\left(\begin{array}{c}
2 i \\
i
\end{array}\right)}{2\left(\begin{array}{c}
m+p \\
p
\end{array}\right)} \\
& \sim \frac{1}{2} \sum_{\gamma=1}^{\infty}\left(\begin{array}{c}
2 \gamma \\
\gamma
\end{array}\right)\left(\frac{\lambda}{(1+\lambda)^{2}}\right)^{\gamma} \\
& =\frac{1}{\lambda-1}=\frac{p}{m-p}
\end{aligned}
$$

if $m / p \rightarrow \lambda>1$.

THEOREM 3.2.

1. $\bar{V}(m, p) / \sqrt{p / 2} \rightarrow \exp \left(\alpha^{2} / 2\right) \int_{\alpha}^{\infty} \exp \left(-t^{2} / 2\right) d t$ if $(m-p) / \sqrt{2 p} \rightarrow \alpha \geq 0$ as $m, p \rightarrow \infty$;

2. $\bar{V}(m, p) / \sqrt{p / 2} \rightarrow 2 \alpha+\exp \left(\alpha^{2} / 2\right) \int_{\alpha}^{\infty} \exp \left(-t^{2} / 2\right) d t$ if $(m-p) / \sqrt{2 p} \rightarrow-\alpha \leq 0$ as $m, p \rightarrow \infty$;

3. For any integer $k, \bar{V}(k+p, p) /(\sqrt{\pi p} / 2) \rightarrow 1$ as $p \rightarrow \infty$.

Proof. By Theorem 2.3, for $m \geq p$,

$$
\bar{V}(m, p)=\sum_{k=0}^{p-1} \frac{\left(\begin{array}{c}
m+p \\
i
\end{array}\right)}{\left(\begin{array}{c}
m+p \\
p
\end{array}\right)}=P(X \leq p-1) / P(X=p),
$$

where $X$ is a binomial random variable with parameters $m+p$ and $1 / 2$. By the central limit theorem [1, p. 42],

$$
\frac{P(X \leq p-1) / P(X=p)}{\sqrt{p / 2}} \rightarrow \exp \left(\alpha^{2} / 2\right) \int_{\alpha}^{\infty} \exp \left(-t^{2} / 2\right) d t
$$

if $(m-p) / \sqrt{2 p} \rightarrow \alpha \geq 0$ as $m, p \rightarrow \infty$. 
By Theorem 2.1, for $m<p, \bar{V}(m, p)=\bar{V}(p, m)+p-m$. Then, by the same argument,

$$
\begin{aligned}
\frac{\bar{V}(m, p)}{\sqrt{p / 2}} & =\frac{(p-m)}{\sqrt{p / 2}}+\frac{\bar{V}(p, m)}{\sqrt{p / 2}} \\
& \rightarrow 2 \alpha+\exp \left(\alpha^{2} / 2\right) \int_{\alpha}^{\infty} \exp \left(-t^{2} / 2\right) d t
\end{aligned}
$$

if $(m-p) / \sqrt{2 p} \rightarrow-\alpha \leq 0$ as $m, p \rightarrow \infty$.

When $\alpha=0, \int_{\alpha}^{\infty} \exp \left(-t^{2} / 2\right) d t=\sqrt{\pi / 2}$. Hence, $\bar{V}(k+p, p) /(\sqrt{\pi p} / 2) \rightarrow 1$ as $p \rightarrow \infty$. The proof of Theorem 3.2 is now complete.

4. The original $(\boldsymbol{m}, \boldsymbol{p})$ urn problem. For any nonnegative integer $m$ and $p$, let $V(m, p)$ be the value of the original $(m, p)$ urn problem proposed by Shepp as stated in section 1. We now want to compare $V(m, p)$ and $\bar{V}(m, p)$.

THEOREM 4.1. $V(m, 0)=\bar{V}(m, 0)$ for all $m=0,1,2, \ldots$ and $V(0, p)=\bar{V}(0, p)=$ $p$ and $V(1, p)=\bar{V}(1, p)=p^{2} /(1+p)$ for all $p=0,1,2, \ldots$

Proof. Since, when $p=0$ or $m=0$ or 1 two problems are the same, they have the same value.

THEOREM 4.2. For any positive integer $m \geq 2$ and $p \geq 1, V(m, p)<\bar{V}(m, p)$.

Proof. For any positive integer $m$ and $p$,

$$
V(m, p)=\max \left\{0, \frac{p-m}{p+m}+\frac{m}{p+m} V(m-1, p)+\frac{p}{p+m} V(m, p-1)\right\}
$$

and

$$
\bar{V}(m, p)=\frac{(p-m)^{+}}{p+m}+\frac{m}{p+m} \bar{V}(m-1, p)+\frac{p}{p+m} \bar{V}(m, p-1) .
$$

By Theorem 4.1, $V(m, 0)=\bar{V}(m, 0)$ for all $m=0,1,2, \ldots$ and $V(1, p)=\bar{V}(1, p)=$ $p^{2} /(p+1)$ for all $p=0,1,2, \ldots$

Now by mathematical induction we can conclude that $\bar{V}(m, p)>V(m, p)$ for any integers $m \geq 2$ and $p \geq 1$ since $\bar{V}(2,1)=1 / 3>V(2,1)=0$.

For the original $(m, p)$ urn problem, if

$$
E(m+1, p)=\frac{m+1}{m+1+p}(-1+V(m, p))+\frac{p}{m+1+p}(1+V(m+1, p-1)) \geq 0,
$$

then $V(m, p)-V(m+1, p) \geq 1 /(m+1+p)$. However, for the acceptance $(m, p)$ urn problem, we do not have this inequality. For instance, $\bar{V}(1,1)-\bar{V}(2,1)=1 / 2-1 / 3=$ $1 / 6<1 / 3$.

In the original $(m, p)$ urn problem, the last ball drawn, under the optimal drawing policy, is always a +1 ball. Similarly, we have the following theorem in the acceptance $(m, p)$ urn problem.

THEOREM 4.3. In the acceptance $(m, p)$ urn problem, the last ball accepted under the optimal acceptance policy is always $a+1$ ball.

Proof. Under the optimal acceptance policy, one will accept the current drawn ball if and only if the number of +1 balls is greater than or equal to the number of -1 balls. Now if the current drawn one is a -1 ball, then the number of +1 balls will be still greater than the number of -1 balls. Hence, the player will accept the 
next drawn ball until he gets a +1 ball. Thus, a -1 ball is never the last accepted ball.

THEOREM 4.4 .

$$
\begin{aligned}
& \lim _{p \rightarrow \infty}(V(m, p+1)-V(m, p))=\lim _{p \rightarrow \infty}(V(m, p)-V(m+1, p))=1, \\
& \lim _{p \rightarrow \infty}(\bar{V}(m, p+1)-\bar{V}(m, p))=\lim _{p \rightarrow \infty}(\bar{V}(m, p)-\bar{V}(m+1, p))=1 .
\end{aligned}
$$

Proof. Since $\lim _{m \rightarrow \infty} \bar{V}(m, p)=0$ for any fixed $p$,

$$
\lim _{p \rightarrow \infty}(\bar{V}(m, p+1)-\bar{V}(m, p))=1+\lim _{p \rightarrow \infty}(\bar{V}(p+1, m)-\bar{V}(p, m))=1 .
$$

Similarly,

$$
\lim _{p \rightarrow \infty}(\bar{V}(m, p)-\bar{V}(m+1, p))=1+\lim _{p \rightarrow \infty}(\bar{V}(p, m)-\bar{V}(p, m+1))=1 .
$$

For any nonnegative integer $m$ and $p$, define

$$
\begin{aligned}
\Delta^{2} V_{p}(m) & =V(m+2, p)+V(m, p)-2 V(m+1, p), \\
\Delta^{2} V_{m}(p) & =V(m, p+2)+V(m, p)-2 V(m, p+1), \\
\Delta^{2} V(m, p) & =V(m+2, p)+V(m, p+2)-2 V(m+1, p+1),
\end{aligned}
$$

and define $\Delta^{2} \bar{V}_{p}(m), \Delta^{2} \bar{V}_{m}(p)$, and $\Delta^{2} \bar{V}(m, p)$ accordingly.

In [4], Chen and Hwang proved that $\Delta^{2} V_{p}(m) \geq 0, \Delta^{2} V_{m}(p) \geq 0$, and $\Delta^{2} V(m, p)$ $\geq 0$. The next theorem shows that $\Delta^{2} \bar{V}_{p}(m)>0, \Delta^{2} \bar{V}_{m}(p)>0$, and $\Delta^{2} \bar{V}(m, p)>0$, for all positive integers $m$ and $p$.

THEOREM 4.5. For any positive integer $m$ and $p, \Delta^{2} \bar{V}_{m}(p)>0, \Delta^{2} \bar{V}_{p}(m)>$ 0 , and $\Delta^{2} \bar{V}(m, p)>0$.

Proof. By definition, $\Delta^{2} \bar{V}_{p}(m)=\bar{V}(m+2, p)+\bar{V}(m, p)-2 \bar{V}(m+1, p)$.

Case 1. Suppose that $m \geq p$; then by Theorem 2.3,

$$
\begin{aligned}
\Delta^{2} \bar{V}_{p}(m)= & 2^{m+p+2} p \int_{0}^{\frac{1}{2}} x^{m+2}(1-x)^{p-1} d x+2^{m+p} p \int_{0}^{\frac{1}{2}} x^{m}(1-x)^{p-1} d x \\
& -2^{m+p+2} p \int_{0}^{\frac{1}{2}} x^{m+1}(1-x)^{p-1} d x \\
= & 2^{m+p} p \int_{0}^{\frac{1}{2}} x^{m}(1-x)^{p-1}\left(4 x^{2}-4 x+1\right) d x>0 .
\end{aligned}
$$

since $m \geq 1$ and $p \geq 1$.

Case 2. Suppose that $p=m+1$; then by Theorems 2.1 and 2.3 ,

$$
\begin{aligned}
\Delta^{2} \bar{V}_{p}(m) & =\bar{V}(m+2, m+1)+\bar{V}(m, m+1)-2 \bar{V}(m+1, m+1) \\
& =\bar{V}(m+2, m+1)+\bar{V}(m+1, m)+1-2 \bar{V}(m+1, m+1) \\
& =\frac{2^{2 m+2}}{\left(\begin{array}{c}
2 m+3 \\
m+1
\end{array}\right)}+\frac{2^{2 m}}{\left(\begin{array}{c}
2 m+1 \\
m
\end{array}\right)}-\frac{2^{2 m+2}}{\left(\begin{array}{c}
2 m+2 \\
m+1
\end{array}\right)} \\
& =2^{2 m+1}(m+1) !(m+1) ! /(2 m+3) !>0 .
\end{aligned}
$$


Case 3. Suppose that $p \geq m+2$; then by Theorems 2.1 and 2.3 ,

$$
\begin{aligned}
\Delta^{2} \bar{V}_{p}(m)=\bar{V}(m+2, p)+\bar{V}(m, p)-2 \bar{V}(m+1, p) \\
=\bar{V}(p, m+2)+\bar{V}(p, m)-2 \bar{V}(p, m+1) \\
=(m+2) 2^{m+p+2} \int_{0}^{\frac{1}{2}} x^{p}(1-x)^{m+1} d x+m 2^{m+p} \int_{0}^{\frac{1}{2}} x^{p}(1-x)^{m-1} d x \\
\quad-(m+1) 2^{m+p+2} \int_{0}^{\frac{1}{2}} x^{p}(1-x)^{m} d x \\
=2^{m+p} \int_{0}^{\frac{1}{2}} x^{p}(1-x)^{m-1}\left(4(m+2)(1-x)^{2}-4(m+1)(1-x)+m\right) d x .
\end{aligned}
$$

Notice that $g(m)=4(m+2)(1-x)^{2}-4(m+1)(1-x)+m$ is strictly increasing in $m$ for all $0 \leq x \leq 1 / 2$ and $g(0) \geq 0$ if $0 \leq x \leq 1 / 2$. Hence,

$$
2^{m+p} \int_{0}^{\frac{1}{2}} x^{p}(1-x)^{m-1}\left(4(m+2)(1-x)^{2}-4(m+1)(1-x)+m\right) d x>0 .
$$

$\Delta^{2} \bar{V}_{m}(p)>0$ and $\Delta^{2} \bar{V}(m, p)>0$ can be proved similarly.

Based on Theorems 2.1, 2.3, and 2.5, we can also prove the following interesting theorems.

THEOREM 4.6. For any nonnegative integer $m$ and $p$,

$$
\begin{aligned}
2 \bar{V}(m, p) & <\bar{V}(m, p)+\bar{V}(m+1, p+1) \\
& <\bar{V}(m+1, p)+\bar{V}(m, p+1) \leq 2 \bar{V}(m+1, p+1) .
\end{aligned}
$$

Proof. By Theorem 2.7, $\bar{V}(m, p)<\bar{V}(m+1, p+1)$, and $2 \bar{V}(m, p)<\bar{V}(m, p)+$ $\bar{V}(m+1, p+1)$.

Case 1 . If $m=0$, then

$$
\begin{aligned}
\bar{V}(m+1, p)+\bar{V}(m, p+1) & =\bar{V}(1, p)+\bar{V}(0, p+1)=p+1+p^{2} /(p+1), \\
\bar{V}(m, p)+\bar{V}(m+1, p+1) & =\bar{V}(0, p)+\bar{V}(1, p+1)=p+(p+1)^{2} /(p+2),
\end{aligned}
$$

and

$$
2 \bar{V}(m+1, p+1)=2 \bar{V}(1, p+1)=2(p+1)^{2} /(p+2) .
$$

It is easy to see that

$$
\bar{V}(m, p)+\bar{V}(m+1, p+1)<\bar{V}(m+1, p)+\bar{V}(m, p+1)<2 \bar{V}(m+1, p+1) .
$$

Case 2. If $p=0$, then

$$
\begin{aligned}
\bar{V}(m, p)+\bar{V}(m+1, p+1) & =\bar{V}(m, 0)+\bar{V}(m+1,1)=1 /(m+2), \\
\bar{V}(m+1, p)+\bar{V}(m, p+1) & =\bar{V}(m+1,0)+\bar{V}(m, 1)=1 /(m+1),
\end{aligned}
$$

and

$$
2 \bar{V}(m+1, p+1)=2 \bar{V}(m+1,1)=2 /(m+2) .
$$


Hence,

$$
\bar{V}(m, p)+\bar{V}(m+1, p+1)<\bar{V}(m+1, p)+\bar{V}(m, p+1) \leq \bar{V}(m+1, p+1) .
$$

Now we assume that $m \geq 1$ and $p \geq 1$.

Case 3. If $m \geq p+1$, then by Theorem 2.3

$$
\bar{V}(m, p)-\bar{V}(m+1, p)=2^{m+p} p \int_{0}^{\frac{1}{2}} x^{m}(1-x)^{p-1}(1-2 x) d x
$$

and

$$
\begin{aligned}
\bar{V}(m, p+1)-\bar{V}(m+1, p+1) \\
\quad=2^{m+p+1}(p+1) \int_{0}^{\frac{1}{2}} x^{m}(1-x)^{p}(1-2 x) d x \\
\quad=2^{m+p}(p+1) \int_{0}^{\frac{1}{2}} x^{m}(1-x)^{p-1}(1-2 x)(2-2 x) d x \\
\quad>2^{m+p} p \int_{0}^{\frac{1}{2}} x^{m}(1-x)^{p-1}(1-2 x) d x .
\end{aligned}
$$

Hence,

$$
\bar{V}(m, p)+\bar{V}(m+1, p+1)<\bar{V}(m+1, p)+\bar{V}(m, p+1) .
$$

On the other hand,

$$
\begin{aligned}
& 2 \bar{V}(m+1, p+1)-\bar{V}(m+1, p)-\bar{V}(m, p+1) \\
& =((m-p) /(m+p+2)) \bar{V}(m, p+1)-((m-p) /(m+p+2)) \bar{V}(m+1, p)>0 .
\end{aligned}
$$

Hence,

$$
\bar{V}(m, p)+\bar{V}(m+1, p+1)<\bar{V}(m+1, p)+\bar{V}(m, p+1)<2 \bar{V}(m+1, p+1) .
$$

Case 4. If $m=p$, then

$$
\bar{V}(m+1, p+1)=\bar{V}(m+1, m+1)=1 / 2 \bar{V}(m+1, m)+1 / 2 \bar{V}(m, m+1) .
$$

Hence,

$$
\bar{V}(m, m)+\bar{V}(m+1, m+1)<2 \bar{V}(m+1, m+1)=\bar{V}(m, m+1)+\bar{V}(m, m+1) .
$$

Case 5. If $m<p$, then

$$
\begin{aligned}
& \bar{V}(m+1, p)+\bar{V}(m, p+1)=\bar{V}(p, m+1)+\bar{V}(p+1, m)+2 p-2 m, \\
& \bar{V}(m, p)+\bar{V}(m+1, p+1)=\bar{V}(p, m)+\bar{V}(p+1, m+1)+2 p-2 m,
\end{aligned}
$$

and

$$
2 \bar{V}(m+1, p+1)=2 \bar{V}(p+1, m+1)+2 p-2 m .
$$

By Case 3,

$$
\bar{V}(m, p)+\bar{V}(m+1, p+1)<\bar{V}(m+1, p)+\bar{V}(m, p+1)<2 \bar{V}(m+1, p+1) .
$$

The proof of Theorem 4.6 is now complete. 
By Theorem 2.5,

$$
\bar{V}(m, p)<\bar{V}(m+1, p+1)<\bar{V}(m+2, p+2)
$$

for all nonnegative integers $m$ and $p$. The next theorem reveals that for all nonnegative integers $m$ and $p, \bar{V}(m+k, p+k)$ is a concave function of $k$.

THEOREM 4.7. For any nonnegative integer $m$ and $p, \bar{V}(m, p)+\bar{V}(m+2, p+2)<$ $2 \bar{V}(m+1, p+1)$.

Proof. By Theorem 2.1,

$\bar{V}(m, p)+\bar{V}(m+2, p+2)-2 \bar{V}(m+1, p+1)=\bar{V}(p, m)+\bar{V}(p+2, m+2)-2 \bar{V}(p+1, m+1)$

if $m<p$. Since it is easy to see that Theorem 4.7 is true when $p=0$, we will assume $m \geq p \geq 1$ in the following proof. Now for any positive integer $m$ and $p$, we write $\bar{V}(m, p)=\bar{V}(n+p, p)$, where $n=m-p \geq 0$. By Theorem 2.3,

$$
\begin{aligned}
\bar{V}(n+p, p) & =2^{n+2 p} p \int_{0}^{\frac{1}{2}} x^{n+p}(1-x)^{p-1} d x \\
& =2^{n} \int_{0}^{\frac{1}{2}} x^{n}(1-x)^{-1} p(4 x(1-x))^{p} d x \\
& =\frac{1}{2} \int_{0}^{1} g(t) p t^{p} d t,
\end{aligned}
$$

where $g(t)=(1-\sqrt{1-t})^{n}(1+\sqrt{1-t})^{-1}(1-t)^{-1 / 2}$. Hence,

$$
\bar{V}(n+p+2, p+2)-2 \bar{V}(n+p+1, p+1)+\bar{V}(n+p, p)=\frac{1}{2} \int_{0}^{1} g(t) h(t) d t,
$$

where $h(t)=(p+2) t^{p+2}-2(p+1) t^{p+1}+p t^{p}$. Notice that $h(t) \geq 0$ if $0 \leq t \leq p /(p+2)$ and $h(t) \leq 0$ if $p /(p+2) \leq t \leq 1$. Also notice that

$$
\int_{0}^{1} h(t) d t=(p+2) /(p+3)-2(p+1) /(p+2)+p /(p+1)<0 .
$$

Hence,

$$
\begin{aligned}
& \bar{V}(n+p+2, p+2)+\bar{V}(n+p, p)-2 \bar{V}(n+p+1, p+1) \\
&=\frac{1}{2} \int_{0}^{1} g(t) h(t) d t=\frac{1}{2} \int_{0}^{t^{*}} g(t) h(t) d t+\frac{1}{2} \int_{t^{*}}^{1} g(t) h(t) d t,
\end{aligned}
$$

where $t^{*}=p /(p+2)$. Hence, by the Mean Value theorem, $1 / 2 \int_{0}^{t^{*}} g(t) h(t) d t=$ $1 / 2 g\left(t_{1}\right) \int_{0}^{t^{*}} h(t) d t$ and $1 / 2 \int_{t^{*}}^{1} g(t) h(t) d t=1 / 2 g\left(t_{2}\right) \int_{t^{*}}^{1} h(t) d t$, where $0<t_{1}<t^{*}<$ $t_{2}<1$. Since $g$ is strictly increasing in $t, 0 \leq t \leq 1,0<g\left(t_{1}\right)<g\left(t_{2}\right)$. Since, $0<\int_{0}^{t^{*}} h(t) d t<-\int_{t^{*}}^{1} h(t) d t, g\left(t_{1}\right) \int_{0}^{t^{*}} h(t) d t<-g\left(t_{2}\right) \int_{h^{*}}^{1} h(t) d t$. Therefore,

$$
\begin{aligned}
\bar{V}(n+p+2, p+2) & +\bar{V}(n+p, p)-2 \bar{V}(n+p+1, p+1) \\
= & \frac{1}{2} g\left(t_{1}\right) \int_{0}^{t^{*}} h(t) d t+\frac{1}{2} g\left(t_{2}\right) \int_{t^{*}}^{1} h(t) d t<0,
\end{aligned}
$$

and the proof of Theorem 4.7 is now complete. 
5. A variation of the acceptance $(\boldsymbol{m}, \boldsymbol{p})$ urn problem. In the stock market, investors try to sell if the future price will go down and try to buy if the future price will go up, so the following variation of the acceptance $(m, p)$ urn problem will be a suitable model.

An urn contains $m$ balls of value -1 and $p$ balls of value +1 . Each turn a ball is drawn randomly, without replacement, and the player decides before the draw whether or not to accept and guess the ball. If he accepts and guesses correctly he gets a +1 ; if he accepts and guesses incorrectly he gets a -1 . The process continues until all $m+p$ balls are drawn.

Let $W(m, p)$ denote the value of this new variation. Let $A_{0}(m, p)$ be the expected value of accepting the current drawn ball from the $(m, p)$ urn and guessing it is a -1 ball, assuming an optimal accepting and guessing policy is followed after the current one. Let $A_{1}(m, p)$ be the expected value of accepting the current drawn ball from the $(m, p)$ urn and guessing it is a +1 ball, assuming an optimal accepting and guessing policy is followed after this one. Let $A(m, p)=\max \left\{A_{0}(m, p), A_{1}(m, p)\right\}$, and let $N(m, p)$ be the expected value of not accepting the current drawn ball from the $(m, p)$ urn, assuming an optimal accepting and guessing policy is followed. It is obvious that $W(m, p)=\max \{A(m, p), N(m, p)\}$. Since $A_{0}(m, p)=(m /(m+p))(1+$ $W(m-1, p))+(p /(m+p))(-1+W(m, p-1))$ and $A_{1}(m, p)=(m /(m+p))(-1+$ $W(m-1, p))+(p /(m+p))(1+W(m, p-1)), A_{0}(m, p)<A_{1}(m, p),=A_{1}(m, p)$, or $>A_{1}(m, p)$ accordingly as $m>p=p$, or $<p$. Hence,

$$
\begin{aligned}
A(m, p) & =(1 /(m+p))(|m-p|+m W(m-1, p)+p W(m, p-1)) \\
& \geq N(m, p)=(1 /(m+p))(m W(m-1, p)+p W(m, p-1)),
\end{aligned}
$$

since $|m-p| \geq 0$. Therefore $W(m, p)=A(m, p)=(1 /(m+p))(|m-p|+m W(m-$ $1, p)+p W(m, p-1))$. The optimal guessing policy is to guess that it is a -1 ball if $m>p$, guess that it is a +1 ball if $m<p$, and guess randomly if $m=p$. If balls of value +1 mean that the price will go up and balls of value -1 mean that the price will go down, then guessing +1 means to buy and guessing -1 means to sell. The optimal guessing policy is consistent with the optimal practice of investors. The following theorems can be proved.

THEOREM 5.1. For any nonnegative integer $i$ and $j, W(i, j)=W(j, i)$.

THEOREM 5.2. For any nonnegative integer $i$ and $j, W(i, j)=\bar{V}(i, j)+\bar{V}(j, i)$.

6. A Bayesian approach to the acceptance $(m, p)$ urn problem. In a financial or marketing problem, the total number of balls is usually known but the number of balls of value -1 is unknown and is a random variable. A Bayesian approach to this optimal stopping problem would be appropriate.

Now let $n=m+p$ be the total number of balls in the urn, and let $\theta$ be the initial prior distribution of the random variable $m$ (number of balls of value -1 ). Let $N_{n}(\theta)$ denote the expected value of not accepting the current drawn ball from the urn, assuming an optimal Bayesian acceptance policy is followed, and let $A_{n}(\theta)$ denote the expected value of accepting the current drawn ball from the urn, assuming an optimal Bayesian acceptance policy is followed. Let $\bar{V}_{n}(\theta)=\max \left\{N_{n}(\theta), A_{n}(\theta)\right\}$ denote the value of the urn with $n$ balls and the prior distribution $\theta$.

Let $x_{1}$ be the value of the first drawn ball. It is easy to see that $A_{n}(\theta)=\int\left(x_{1}+\right.$ $\left.\bar{V}_{n-1}\left(\theta\left(x_{1}\right)\right)\right) \theta\left(d x_{1}\right)$ and $N_{n}(\theta)=\int \bar{V}_{n-1}\left(\theta\left(x_{1}\right)\right) \theta\left(d x_{1}\right)$. Here $\theta\left(x_{1}\right)$ is the posterior distribution of the number of balls of value -1 after the first draw given that $X_{1}=x_{1}$. Since $A_{n}(\theta) \geq N_{n}(\theta)$ if and only if $\int x_{1} \theta\left(d x_{1}\right)=\theta\left(X_{1}=1\right)-\theta\left(X_{1}=-1\right) \geq 0$, one would accept the current drawn ball if $\theta\left(X_{1}=1\right) \geq \theta\left(X_{1}=-1\right)$. Therefore, the 
optimal Bayesian acceptance policy can be simply stated as follows: for $k=1,2, \ldots, n$, the player will accept the $k$ th drawn ball if and only if $\theta\left(X_{k}=1 \mid x_{1}, x_{2}, \ldots, x_{k-1}\right) \geq$ $\theta\left(X_{k}=-1 \mid x_{1}, x_{2}, \ldots, x_{k-1}\right)$ where $\theta\left(\cdot \mid x_{1}, \ldots, x_{k-1}\right)$ is the posterior distribution of the number of -1 balls given that $X_{1}=x_{1}, X_{2}=x_{2}, \ldots, X_{k-1}=x_{k-1}$.

Now suppose that the initial prior distribution $\theta$ of $m$ (the number of -1 balls) is uniform over the set $\{0,1,2, \ldots, n\}$. Since $\sum_{i=1}^{k} X_{i}$ is a sufficient statistic for the unknown parameter $m, \theta\left(X_{k}=1 \mid \sum_{i=1}^{k-1} X_{i}\right) \geq \theta\left(X_{k}=-1 \mid \sum_{i=1}^{k-1} X_{i}\right)$ if an only if $\sum_{i=1}^{k-1} X_{i} \geq 0$. The player will accept the $k$ th drawn ball if and only if $\sum_{i=1}^{k-1} X_{i} \geq 0$. It is worth noticing that the character of the optimal Bayesian acceptance policy is similar to that of the optimal acceptance policy of the non-Bayesian urn problem. However, when $m$ is known, under the optimal acceptance policy the ball accepted last is always a +1 , but under an optimal Bayesian acceptance policy the ball accepted last is always a -1 except for the $n$th ball.

The following are values of $\bar{V}_{n}(\theta)$ when $\theta$ is uniform:

$$
\begin{array}{llrl}
n & =1, & & \bar{V}_{n}(\theta)=0, \\
n & =2, & & \bar{V}_{n}(\theta)=1 / 6, \\
n & =3, & & \bar{V}_{n}(\theta)=1 / 3, \\
n & =4, & & \bar{V}_{n}(\theta)=17 / 30 .
\end{array}
$$

Notice that $E(m \mid n=2)=1$, but $\bar{V}_{2}(\theta)=1 / 6<\bar{V}(1,1)=1 / 2 ; E(m \mid$ $n=4)=2$, but $\bar{V}_{4}(\theta)=17 / 30<\bar{V}(2,2)=5 / 6$. These facts are expected since we have full information about an acceptance $(m, p)$ urn and we have only partial information about a random acceptance $(m, p)$ urn, i.e., when $m$ is a random variable. Furthermore, $\bar{V}_{n}(\theta)$ is nondecreasing in $n$ since the player has more times to decide whether or not to accept.

7. Application and numerical illustration. The acceptance $(m, p)$ urn model studied above can be useful in the following financial situation. Suppose that we expect there will be $m$ downs and $p$ ups in the stock price (or bond price). Suppose that the up or down will be on an equal scale. We buy the stock and sell it at the next time unit. If the price goes up one unit we make a profit; otherwise we lose. Our goal is to maximize the gain. Based on our acceptance $(m, p)$ urn model, we should buy the stock if and only if the number of the ups is greater than the number of the downs. Otherwise we should not have any trading.

The variation of the acceptance $(m, p)$ urn model discussed in section 5 can be used in the following situation. Suppose that we expect that there will be $m$ downs and $p$ ups in the stock price. If we know the price will be up, certainly we should buy the stock and sell later. If we know the price will be down, we should sell the stock and buy back later. Our goal is to maximize the gain between "in and out." The optimal strategy will be that "buy now sell later" if the number of the ups is greater than the number of the downs; conversely, "sell now and buy back later" if the number of the ups is less than the number of the downs.

Certainly, the numbers of the ups and downs are not known, and they are random. Therefore, the Bayesian approach to the acceptance $(m, p)$ urn model would be much more suitable to the financial application. The details will be presented in another article.

The following three tables of values of $V(m, p), \bar{V}(m, p)$, and $W(m, p)$ are given for the sake of comparison.

Acknowledgment. We would like to thank the referee for his invaluable comments which led to a simpler and more intuitive proof of Theorem 2.3, and also for correcting a mistake in Theorem 3.2. 
TABLE 1

$V(m, p)$

\begin{tabular}{|c|cccccccccc|}
\hline$p$ (plus) & \multicolumn{10}{|c|}{$m$ (minus) } \\
\cline { 2 - 11 } & 0 & 1 & 2 & 3 & 4 & 5 & 6 & 7 & 8 & 9 \\
\hline \hline 9 & 9 & 8.10 & 7.20 & 6.31 & 5.43 & 4.58 & 3.75 & 2.95 & 2.21 & $\underline{1.53}$ \\
8 & 8 & 7.11 & 6.22 & 5.35 & 4.49 & 3.66 & 2.86 & 2.11 & $\underline{1.43}$ & 0.84 \\
7 & 7 & 6.13 & 5.25 & 4.39 & 3.56 & 2.76 & 2.01 & $\underline{1.34}$ & 0.66 & 0.23 \\
6 & 6 & 5.14 & 4.29 & 3.45 & 2.66 & 1.91 & $\underline{1.23}$ & 0.66 & 0.23 & 0 \\
5 & 5 & 4.17 & 3.33 & 2.54 & 1.79 & $\underline{1.12}$ & 0.55 & 0.15 & 0 & 0 \\
4 & 4 & 3.20 & 2.40 & 1.66 & $\underline{1.00}$ & 0.44 & 0.07 & 0 & 0 & 0 \\
3 & 3 & 2.25 & 1.50 & $\underline{0.85}$ & 0.34 & 0 & 0 & 0 & 0 & 0 \\
2 & 2 & 1.33 & $\underline{0.67}$ & 0.20 & 0 & 0 & 0 & 0 & 0 & 0 \\
1 & 1 & $\underline{0.50}$ & 0 & 0 & 0 & 0 & 0 & 0 & 0 & 0 \\
0 & $\underline{0}$ & 0 & 0 & 0 & 0 & 0 & 0 & 0 & 0 & 0 \\
\hline
\end{tabular}

TABLE 2

$\bar{V}(m, p)$.

\begin{tabular}{|c|cccccccccc|}
\hline \multirow{2}{*}{$p$ (plus) } & \multicolumn{10}{|c|}{$m$ (minus) } \\
\cline { 2 - 11 } & 0 & 1 & 2 & 3 & 4 & 5 & 6 & 7 & 8 & 9 \\
\hline \hline 9 & 9 & 8.10 & 7.22 & 6.36 & 5.53 & 4.73 & 3.99 & 3.30 & 2.70 & $\underline{2.20}$ \\
8 & 8 & 7.11 & 6.24 & 5.41 & 4.60 & 3.85 & 3.16 & 2.55 & $\underline{2.05}$ & 1.70 \\
7 & 7 & 6.13 & 5.28 & 4.47 & 3.70 & 3.00 & 2.39 & $\underline{1.89}$ & 1.55 & 1.30 \\
6 & 6 & 5.14 & 4.32 & 3.55 & 2.83 & 2.22 & $\underline{1.72}$ & 1.39 & 1.16 & 0.99 \\
5 & 5 & 4.17 & 3.38 & 2.66 & 2.03 & $\underline{1.53}$ & 1.22 & 1.00 & 0.85 & 0.73 \\
4 & 4 & 3.20 & 2.47 & 1.83 & $\underline{1.33}$ & 1.03 & 0.83 & 0.70 & 0.60 & 0.53 \\
3 & 3 & 2.25 & 1.60 & $\underline{1.10}$ & 0.83 & 0.66 & 0.55 & 0.47 & 0.41 & 0.36 \\
2 & 2 & 1.33 & $\underline{0.83}$ & 0.60 & 0.47 & 0.38 & 0.32 & 0.28 & 0.24 & 0.22 \\
1 & 1 & $\underline{0.50}$ & 0.33 & 0.25 & 0.20 & 0.17 & 0.14 & 0.13 & 0.11 & 0.10 \\
0 & $\underline{0}$ & 0 & 0 & 0 & 0 & 0 & 0 & 0 & 0 & 0 \\
\hline
\end{tabular}

TABLE 3

$W(m, p)$.

\begin{tabular}{|c|cccccccccc|}
\hline \multirow{2}{*}{$p$ (plus) } & \multicolumn{10}{|c|}{$m$ (minus) } \\
\cline { 2 - 11 } & 0 & 1 & 2 & 3 & 4 & 5 & 6 & 7 & 8 & 9 \\
\hline \hline 9 & 9 & 8.20 & 7.44 & 6.72 & 6.06 & 5.46 & 4.98 & 4.60 & 4.40 & $\underline{4.40}$ \\
8 & 8 & 7.22 & 6.48 & 5.82 & 5.20 & 4.70 & .4 .32 & 4.10 & $\underline{4.10}$ & 4.40 \\
7 & 7 & 6.26 & 5.56 & 4.94 & 4.40 & 4.00 & 3.78 & $\underline{3.78}$ & 4.10 & 4.60 \\
6 & 6 & 5.28 & 4.64 & 4.10 & 3.66 & 3.44 & $\underline{3.44}$ & 3.78 & 4.32 & 4.98 \\
5 & 5 & 4.34 & 3.76 & 3.32 & 3.06 & $\underline{3.06}$ & 3.44 & 4.00 & 4.70 & 5.46 \\
4 & 4 & 3.40 & 2.94 & 2.66 & $\underline{2.66}$ & 3.06 & 3.66 & 4.40 & 5.20 & 6.06 \\
3 & 3 & 2.50 & 2.20 & $\underline{2.20}$ & 2.66 & 3.32 & 4.10 & 4.94 & 5.82 & 6.72 \\
2 & 2 & 1.66 & $\underline{1.66}$ & 2.20 & 2.94 & 3.76 & 4.64 & 5.56 & 6.48 & 7.44 \\
1 & 1 & $\underline{1.00}$ & 1.66 & 2.50 & 3.40 & 4.34 & 5.28 & 6.26 & 7.22 & 8.20 \\
0 & $\underline{0}$ & 1 & 2 & 3 & 4 & 5 & 6 & 7 & 8 & 9 \\
\hline
\end{tabular}

\section{REFERENCES}

[1] P. Billingsley, Convergence of Probability Measures, John Wiley and Sons, New York, 1968.

[2] W. M. BoycE, Stopping rules for selling bonds, Bell J. Econ. Manage Sci., 1 (1970), pp. $27-53$.

[3] W. M. Boyce, On a simple optimal stopping problem, Discrete Math., 5 (1973), pp. $297-312$.

[4] R. W. Chen And F. K. Hwang, On the values of an ( $m, p)$ urn, Congr. Numer., 41 (1984), pp. $75-84$.

[5] H. W. Gould, Combinatorial Identities, Morgantown, WV, 1972.

[6] N. L. Johnson And S. Kotz, Urn Models and Their Applications, John Wiley and Sons, New York, 1977.

[7] L. A. SHEPP, Explicit solutions to some problems of optimal stopping, Ann. Math. Statist., 40 (1969), pp. 993-1010. 